\title{
Calculated Threshold Currents of Nitride- and Phosphide-Based Quantum-Well Lasers
}

\author{
P. Rees, C. Cooper, P. M. Smowton, P. Blood, and J. Hegarty
}

\begin{abstract}
We have calculated the room temperature gain-current characteristics for a $360 \mathrm{~nm}$ wavelength, $80 \AA$ GaN- $\mathbf{A l}_{0.14} G_{0.86} \mathbf{N}$ and a red-emitting, $80 \AA \mathbf{G a}_{0.51} \mathbf{I n}_{0.49} \mathbf{P}-$ $\left(\mathbf{A l}_{0.44} \mathbf{G a}_{0.56}\right)_{0.51} \mathbf{I n}_{0.49} \mathbf{P}$ quantum well laser structures, including many body effects. Although the carrier density and spontaneous current are much higher (by a factor of 4 and 3 , respectively) in the nitride structures for a given local gain, the higher confinement factor at short wavelengths means the intrinsic threshold current of these devices is predicted to be approximately twice that of red lasers with the same optical loss.
\end{abstract}

$\mathbf{T}$

THE III-V nitride material systems are attracting much attention for their potential as optoelectronic devices at blue and ultraviolet wavelengths. Recent advances in growth procedures have allowed $\mathrm{GaN}-\mathrm{AlGaN}$ quantum well structures to be grown [1] and $\mathrm{GaN}$ to be deposited on a variety of substrates with an improving crystalline quality (for a review see reference [2]). Although some work has been published on optical gain in bulk material, as yet very little work has been reported on the gain-current characteristics of quantum well devices. In this letter we report results of calculations of the gain-current characteristics for a $80 \AA \mathrm{GaN}-\mathrm{Al}_{0.14} \mathrm{Ga}_{0.86} \mathrm{~N}$ quantum well, emitting at approximately $360 \mathrm{~nm}$ and compare the results with those of a similar structure in the AlGaInP material system, emitting in the red, to provide a contrast for assessing the feasibility of such a device. The AlGaInP material system is chosen for comparison having the largest bandgap of well-understood III-V semiconductors.

There are a number of fundamental differences between lasers at short wavelengths in the blue and present-day devices operating in the red and near infra-red. The carrier density needed to achieve transparency is significantly higher due to the higher electron and hole effective masses, and the radiative recombination matrix element, which is inversely proportional to the material band gap, is smaller than in narrower gap semiconductors. Although the lower matrix element reduces the recombination rate per carrier, the higher threshold carrier density leads to a higher intrinsic recombination current at a given local gain. Finally we note that as the wavelength is

Manuscript received July 18, 1995; revised October 27, 1995. P. Rees was supported in part by the EC under the Human Capital Mobility research training program. C. Cooper and P. M. Smowton were supported in part by the EPSRC.

P. Rees and J. Hegarty are with the Physics Department, Trinity College, Dublin 2, Ireland.

C. Cooper, P. M. Smowton, and P. Blood are with the Department of Physics and Astronomy, University of Wales Cardiff, P.O. Box 913, Cardiff CF2 3YB, UK.

Publisher Item Identifier S 1041-1135(96)01259-1. reduced the confinement factor of a quantum well of a given width increases thus reducing the gain requirement for a laser of given cavity length and facet reflectivity. All these factors suggest that the performance expected of a III-V blue laser is not a simple extrapolation from red-emitting devices operating at a longer wavelength.

In this paper we compare threshold currents of nitride and phosphide lasers, both calculated including many-body effects, and discuss optimisation of the cavity length of these devices. Khan et al. [1] have observed photoluminescence from $\mathrm{GaN}-\mathrm{Al}_{0.14} \mathrm{Ga}_{0.86} \mathrm{~N}$ quantum wells (on sapphire substrates) of various widths and we will use this well structure for our calculations. All computations are for a hypothetical, unstrained, cubic structure due to the lack of detailed band structure information and strain parameters in $\mathrm{GaN}$. We expect the effects of strain to be similar to that in other material systems therefore our comparison of unstrained structures should give a good indication of the important distinctions between the short and longer wavelength devices.

Previously we have shown that the inclusion of many body effects is an essential consideration in the calculation of gain in the $\mathrm{GaN}$ material system [3], nevertheless our calculations suggest that excitons are screened-out at typical carrier densities in a laser and do not contribute significantly to the gain in GaN so we use a model in which Coulomb enhancement is included in recombination from an electron hole plasma. Coulomb enhancement has not been included in previous calculations for phosphide-based lasers and to enable us to make meaningful comparisons we include it here for the first time. We calculate the many-body gain using the matrix inversion method to solve the equation for the intraband polarisation [4]. The calculation uses parabolic subbands, and strict $k$ selection is adopted for optical transitions as we are considering an undoped active region with the optical transition matrix element as described by Kane [5]. The dephasing time, which we assume to be given by the carrier-carrier scattering lifetime, is calculated as described in reference [6]; we have not included carrier-phonon scattering. The masses for the electron and heavy hole in GaN were taken as 0.2 and 0.8 respectively [7]. Our calculations ignore the light hole band because we lack any value for its effective mass. We have used a fixed ratio of $0.7: 0.3$ conduction: valence band offset ratio [8]. The calculations for both material systems are identical though the resulting Coulomb enhancement is smaller in GaInP.

Fig. 1 shows the calculated TE peak local gain $(g)$ versus carrier clensity $(n)$ for an $80 \AA \mathrm{GaN}-\mathrm{Al}_{0.14} \mathrm{Ga}_{0.86} \mathrm{~N}$ 


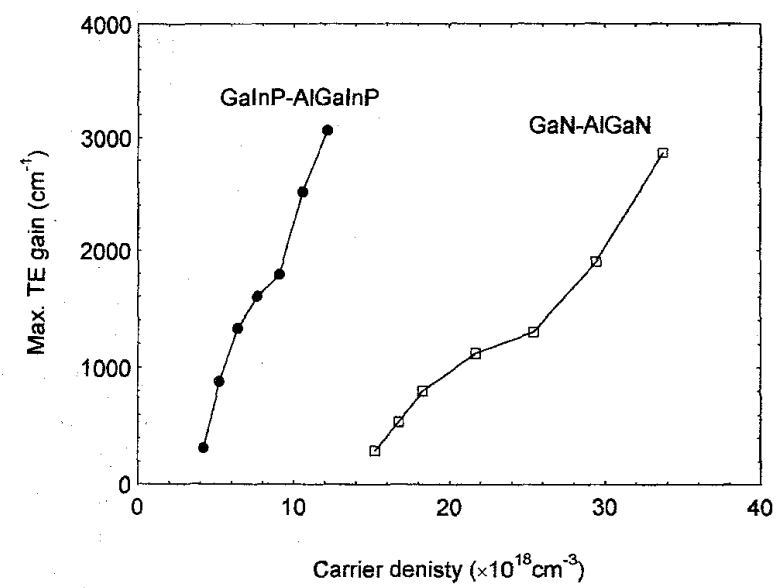

Fig. 1. Peak TE gain versus carrier density calculated for an 80 $\AA \mathrm{GaN}-\mathrm{Al}_{0.14} \mathrm{Ga}_{0.86} \mathrm{~N}$ and an $80 \AA\left(\mathrm{Al}_{0.44} \mathrm{Ga}_{0.56}\right)_{0.5 \mathrm{I}} \mathrm{In}_{0.49} \mathrm{P}-$ $\mathrm{Ga}_{0.51} \operatorname{In}_{0.49} \mathrm{P}$ quantum well, including Coulomb enhancement in both cases.

quantum well and for an unstrained, red-emitting $80 \AA$ $\mathrm{Ga}_{0.51} \mathrm{In}_{0.49} \mathrm{P}-\left(\mathrm{Al}_{0.44} \mathrm{Ga}_{0.56}\right)_{0.51} \mathrm{In}_{0.49} \mathrm{P}$ quantum well chosen because, with the band offset ratio of $0.7: 0.3$ [9], both structures have similar conduction and valence band well depths. (For convenience, $n$ is expressed per unit volume and is calculated as the carrier density per unit area multiplied by the well thickness.) The calculation for the phosphide structure used electron and hole effective masses of 0.11 and $0.43 m_{0}$, the light holes being omitted from the calculation as for the $\mathrm{GaN}$ well to give a more meaningful comparison. The values of the dielectric constant for $\mathrm{GaN}$ and $\mathrm{GaInP}$ were 9.5 [2] and 11.75 [9] respectively.

It is clear from Fig. 1 that the gain due to the lowest e1-hh1 transition saturates at a lower value for the GaN well; this is because the matrix element for optical transitions is inversely proportional to the bandgap and the electron effective mass [7]. This could cause devices having a high cavity loss to operate on a higher order transition $(n>1)$ to achieve the high level of gain required, leading to a shift in wavelength and higher threshold current. The carrier density required to achieve the same local gain is a factor of 4 higher for the GaN well compared with the GaInP well.

We have obtained the spontaneous recombination current by integrating the spontaneous emission spectrum derived from the absorption spectrum using detailed balance arguments [10]. Plots of the calculated peak TE gain verses spontaneous recombination current per unit area $(J)$ for a $80 \AA\left(\mathrm{Al}_{0.44} \mathrm{Ga}_{0.56}\right)_{0.51} \mathrm{In}_{0.49} \mathrm{P}-\mathrm{Ga}_{0.51} \mathrm{In}_{0.49} \mathrm{P}$ and a $80 \AA$ $\mathrm{GaN}-\mathrm{Al}_{0.14} \mathrm{Ga}_{0.86} \mathrm{~N}$ quantum well are shown in Fig. 2. The transparency current densities are 390 and $1210 \mathrm{Acm}^{-2}$ respectively, and expressing these curves in a logarithmic form $[11,12]$ the scaling constants are $J_{t}=830$ and 2030 A $\mathrm{cm}^{-2}$, and $g_{t}=1490$ and $1200 \mathrm{~cm}^{-1}$, respectively. For a typical threshold gain of $1250 \mathrm{~cm}^{-1}$ the value of the spontaneous recombination current is $750 \mathrm{Acm}^{-2}$ for GaInP and $2100 \mathrm{Acm}^{-2}$ for $\mathrm{GaN}$, a factor of 3 greater. The carrier densities required to achieve this gain are $6 \times 10^{18} \mathrm{~cm}^{-3}$ and $24 \times 10^{18} \mathrm{~cm}^{-3}$ for GaInP and GaN respectively (Fig. 1); the

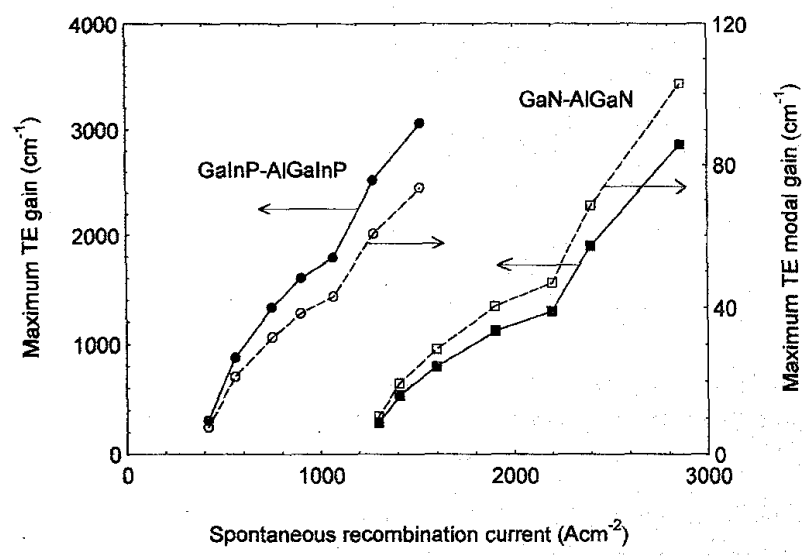

Fig. 2. Peak TE gain (solid lines) and peak modal TE gain (dotted lines) versus spontaneous recombination current calculated for a $80 \AA$ GaN-Alo.14 Ga0.86 $\mathrm{N}$ and a $80 \AA\left(\mathrm{Al}_{0.44} \mathrm{Ga}_{0.56}\right)_{0.51} \mathrm{In}_{0.49} \mathrm{P}-\mathrm{Ga}_{0.51} \mathrm{In}_{0.49} \mathrm{P}$ quantum well, including Coulomb enhancement.

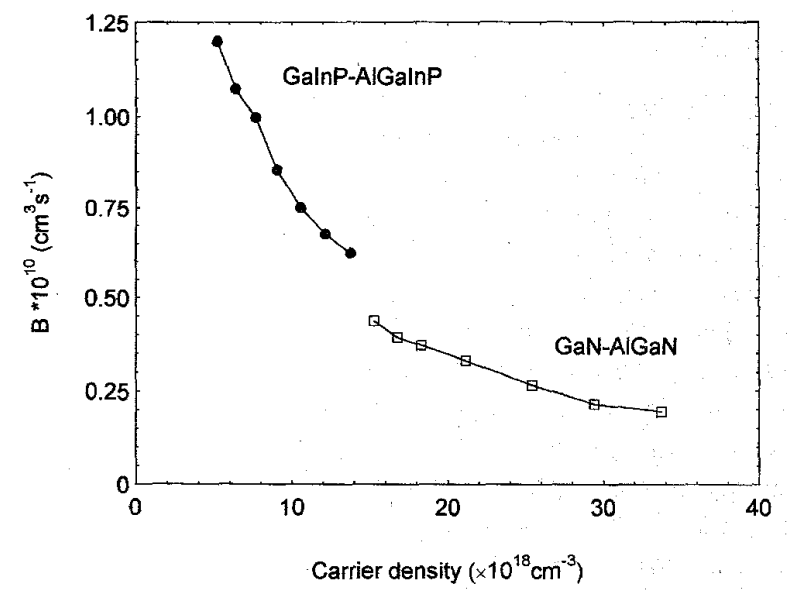

Fig. 3. The radiative recombination coefficient, $B$, calculated for an $80 \AA$ GaN-Al ${ }_{0.14} \mathrm{Ga}_{0.86} \mathrm{~N}$ and a $80 \AA\left(\mathrm{Al}_{0.44} \mathrm{Ga}_{0.56}\right)_{0.51} \mathrm{In}_{0.49} \mathrm{P}-\mathrm{Ga}_{0}{ }_{51} \mathrm{In}_{0.49} \mathrm{P}$ quantum well over the carrier densities given in Fig. 1.

ratio of threshold currents is reduced compared to the carrier densities by the lower matrix element in the nitrides.

The spontaneous recombination current per unit volume is often approximated by the relation $J_{\text {spon }}=e B n p$, where $n$ and $p$ are the electron and hole volume densities $(n=p$ in an undoped active region) and $B$ is the radiative recombination coefficient. In Fig. 3 we show the values of $B$ obtained from the results of the calculation for the values of carrier density shown in Fig. 1. For the carrier densities quoted above the corresponding values of $B$ are $1.10 * 10^{-10}$ and $0.29 * 10^{-10} \mathrm{~cm}^{3} \mathrm{~s}^{-1}$ for GaInP and GaN respectively. As expected the value of $B$ is lower in the nitride well because of the smaller matrix element, though the dependence upon carrier density is not as strong as in the phosphide structure.

From Fig. 2, it is clear that to achieve a specific value of local gain the spontaneous recombination current is significantly higher for the GaN well. However a more useful comparison is the threshold current for lasers with the same optical loss, i.e., the current for the same modal gain, $G$ : $(G=(\Gamma g)$, where $\Gamma$ is 
the optical confinement factor). A typical waveguide cladding in AlGaInP lasers is $\left(\mathrm{Al}_{0.70} \mathrm{Ga}_{0.30}\right)_{0.51} \mathrm{In}_{0.49} \mathrm{P}$ [13] giving an optimum waveguide width of $235 \mathrm{~nm}$ and $\Gamma=0.024$ per well at an operating wavelength of $650 \mathrm{~nm}$ (using refractive indices of 3.35 and 3.26 [14]). Although data is not available for the refractive indices of the cubic phase of AlGaN, an index step of 0.09 should be possible since the refractive indices obtainable for different compositions are proportional to the band gaps available [15] and the range of band gaps available from the cubic phase of $\mathrm{AlGaN}$ is significantly greater than from AlGaInP. For $\mathrm{AlGaN}$, the optimised waveguide width is $149 \mathrm{~nm}$ for a similar waveguide step giving $\Gamma=0.036$ per well, which is considerably greater than for the AlGaInP structure as the $80 \AA \mathrm{AlGaN}$ well overlaps with more of the lowest optical mode at shorter wavelengths and less local gain is required to overcome the cavity losses. Fig. 2 shows a plot of the calculated modal gain versus spontaneous current density.

For a modal gain of $40 \mathrm{~cm}^{-1}$ the threshold current densities are 940 and $1870 \mathrm{Acm}^{-2}$ for the phosphide and nitride devices respectively, a ratio of about a factor 2 . The effect of the higher confinement factor is significant in moderating the increase in threshold current of the nitrides. From the gain current curves it is usually possible to estimate the cavity lengths for an optimum threshold current assuming the first pair of subbands are providing the gain [11], giving values of about 330 $\mu \mathrm{m}$ (phosphide) and $270 \mu \mathrm{m}$ (nitride) (for a facet reflectivity of 0.3 and scattering loss of $15 \mathrm{~cm}^{-1}$ ), however both of these lengths result in local gain requirements which cannot be met from these sub-bands alone. If operation on the lowest sub-bands is required then longer devices are necessary and the optimum design is that which utilises the maximum gain available from this transition; for the nitrides the maximum modal gain from the $n=1$ transition is about $45 \mathrm{~cm}^{-1}$. For the parameters used above these translate into lengths of about $425 \mu \mathrm{m}$ (phosphide) and $380 \mu \mathrm{m}$ (nitride). Note that since the transparency current density for the nitrides is about 1200 $\mathrm{Acm}^{-2}$, more than half the total current, there is no advantage to be gained by increasing the number of wells because the doubling of the total current to reach transparency is not balanced by a decrease in the current required to overcome the optical losses. All these comments refer to an ideal device and for real devices these threshold currents must be scaled by the internal quantum efficiency. If there is a significant thermally activated leakage current which is sensitive to the position of the quasi-Fermi levels then it may be desirable to increase the number of wells to lower the local gain requirement per well (see, e.g., [16]). Similarly, a multiple well laser may be desirable to maintain the lasing process on the lowest pair to sub-bands.

In summary, detailed calculations including many-body Coulomb enhancement show that nitride quantum well lasers at about $360 \mathrm{~nm}$ wavelength have significantly higher threshold carrier and current densities than longer wavelength de- vices, however due to the higher confinement factor at short wavelength the threshold current density for a given cavity loss is only about twice that of a phosphide $650 \mathrm{~nm}$ laser. Our calculations suggest that, for nitride lasers, operation on the lowest pair of sub-bands is only possible for devices requiring a modal gain less than about $45 \mathrm{~cm}^{-1}$, and this consideration may dictate the choice of length rather than the more usual optimisation process. The transparency current is the major contribution to the total intrinsic threshold current in nitride devices so there is no direct advantage in using more than one well, however multiple well devices may be necessary to retain operation on the $n=1$ sub-bands and to reduce extrinsic leakage currents.

\section{ACKNOWLEDGMENT}

The authors would like to thank Dr. I. Galbraith and Prof. W. W. Chow for help and useful discussions.

\section{REFERENCES}

[1] M. A. Khan, R. A. Skogman, and J. M. Van Hove, "Photoluminescence characteristics of AlGaN-GaN-AlGaN quantum wells," Appl. Phys. Lett., vol. 56, pp. 1257-1259, 1990.

[2] S. Strite and H. Morkoc, "GaN, AlN, and InN: A review," J. Vac. Sci. Technol. B, vol. 10, pp. 1237-1266, 1992.

[3] P. Rees, C. Cooper, P. Blood, P. M. Smowton, and J. Hegarty, "Gain characteristics of GaN quantum wells including many-body effects," Electron Lett., accepted for publication.

[4] H. Haug and S. Koch, Quantum Theory of the Optical and Electronic Properties of Semiconductors. Singapore: World Scientific, 1990, p. 212 ,

[5] E. Kane, "Thomas-Fermi approach to impure semiconductor band structure," Phys. Rev., vol. 131, pp. 79-88, 1963.

[6] R. A. H. Hamilton and P. Rees, "Line broadening due to carrier-carrier scattering in quantum well heterostructures," Semiconduct. Sci. Technol., vol. 8, pp. 728-734, 1993.

[7] D. Ahn, "Qualitative estimation of optical gain in wide-band-gap semiconductor quantum wells," J. Appl. Phys., vol. 76, pp. 8206-8208, 1994.

[8] G. Martin, S. Strite, A. Botchkarev, A. Agarwal, A. Rockett, H. Morkoc, W. R. L. Lambrecht, and B. Segall, "Valence-band discontinuity between GaN and AllN measured by X-ray photoemission spectroscopy," Appl. Phys. Lett., vol. 65, pp. 610-612, 1994.

[9] C. T. H. F. Liedenbaum, A. Valster, A. L. G. J. Severens, and G.W. 't Hooft, "Determination of the GaInP/AlGaInP Band offset," Appl. Phys. Lett., vol. 57, no. 25, pp. 2698-2790, 1990.

[10] C. H. Henry, R. A. Logan, and F. R. Meritt, "Measurement of gain and absorption in AlGaAs buried heterostructure lasers," J. Appl. Phys., vol. 51, pp. 3042-3050.

[11] P. W. A. McIlroy, A. Kurobe, and Y. Uematsu, "Analysis and application of theoretical gain curves to the design of multi-quantum-well lasers," IEEE J. Quantum Electron, QE-21, pp. 1958-1963, 1985.

[12] P. Blood, in Physics and Technology of Hetrostructure Devices, D. V. Morgan and R. H. Williams, Eds. Stevenage, UK: Peter Perigrinus, 1991, ch. 7

[13] H. D. Summers, P. Blood, and P. Rees, "Gain-current characteristics of strained AlGaInP quantum well lasers," Appl. Phys. Lett., vol. 63, no. 20, pp. 2792-2794, 1993.

[14] Y. Kaneko and $\mathrm{K}$. Kishino, "Refractive indices measurement of $(\mathrm{GaInP})_{m} /(\mathrm{AlInP})_{n}$ quasi-quaternaries and GaInP/AlInP multiple quantum wells," J. Appl. Phys., vol. 76, 3, pp. 1809-1818, 1994.

[15] M. A. Afromowitz, "Refractive index of $\mathrm{Ga}_{1-x} \mathrm{Al}_{x} \mathrm{As}$," Solid State Communications, vol. 15, pp. 59-63, 1974.

[16] P. M. Smowton and P. Blood, "Optimisation of $670 \mathrm{~nm}$ strained quantum well laser diodes for high temperature operation," IEE Proc. Optoelectronics, vol. 141, pp. 136-140, 1994. 